\title{
ASTE observations of dense molecular gas in galaxies
}

\author{
K. Kohno ${ }^{1}$, K. Muraoka ${ }^{1}$, K. Nakanishi ${ }^{2}$, T. Tosaki ${ }^{2}$, N. Kuno ${ }^{2}$,
}

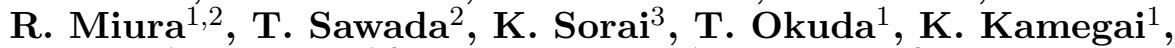
K. Tanaka ${ }^{1}$, A. Endo ${ }^{1,2}$, B. Hatsukade ${ }^{1}$, H. Ezawa ${ }^{2}$, S. Sakamoto ${ }^{2}$, J. Cortes $^{2,4}$, N. Yamaguchi ${ }^{2}$, H. Matsuo ${ }^{2}$ and R. Kawabe ${ }^{2}$

\author{
${ }^{1}$ University of Tokyo \\ email: kkohno@ioa.s.u-tokyo.ac.jp \\ ${ }^{2}$ National Astronomical Observatory of Japan \\ ${ }^{3}$ Hokkaido University \\ ${ }^{4}$ University of Chile
}

\begin{abstract}
Atacama Submillimeter Telescope Experiment (ASTE) is a joint project between Japan and Chile for installing and operating a $10 \mathrm{~m}$ high precision telescope in the Atacama Desert in order to explore the southern sky through the submillimeter wavelength. We have achieved an accuracy of $19 \mu \mathrm{m}$ (rms) for the main reflector surface and a stable radio pointing accuracy of about 2 arcsec (rms). A $350 \mathrm{GHz}$ cartridge type SIS mixer receiver achieves good performance with a typical system noise temperature of $150 \sim 250 \mathrm{~K}$ in DSB and a main beam efficiency of $0.6 \sim 0.7$ during winter nights.

A large scale $\mathrm{CO}(3-2)$ imaging survey of nearby galaxies using ASTE is now in progress. One of our goals is to compare our wide area $\mathrm{CO}(3-2)$ images with existing $\mathrm{CO}(1-0)$ data as well as distributions of massive star formation tracers (i.e., $\mathrm{H} \alpha$ and radio continuum emission) in order to understand the physical mechanism which controls the global star formation properties such as star formation efficiency. Initial CO(3-2) maps of some sample galaxies (M 83, NGC 604 in M 33, NGC 1672, \& NGC 7130) are reported.
\end{abstract}

Keywords. galaxies: ISM, galaxies: starburst, submillimeter, telescopes
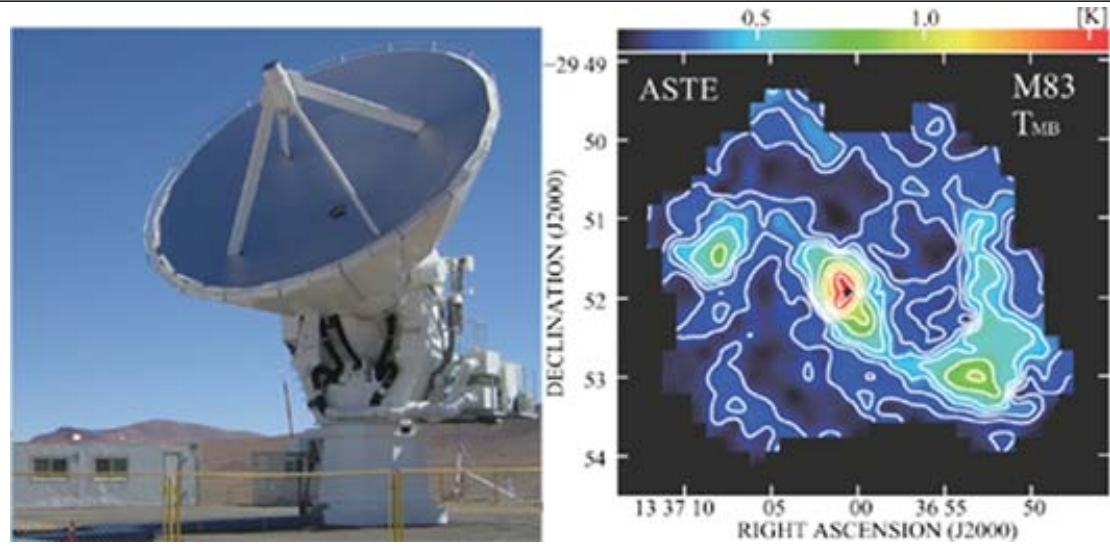

Figure 1. (left) ASTE observatory at Pampa la Bola $(4860 \mathrm{~m})$ in the Atacama desert, Chile. (right) A CO(3-2) peak temperature map of M83 taken with ASTE (Muraoka et al. 2006).

\section{References}

Muraoka, K., et al. 2006, PASJ submitted

Tosaki, T., et al. 2006, this volume 